\title{
Fault-Tolerant Parity Readout on a Shuttling-Based Trapped-Ion Quantum Computer
}

\author{
J. Hilder®, ${ }^{1}$ D. Pijn $\odot,{ }^{1}$ O. Onishchenko $\odot,{ }^{1}$ A. Stahl, ${ }^{1}$ M. Orth, ${ }^{1}$ B. Lekitsch $\odot,{ }^{1}$ A. Rodriguez-Blanco, ${ }^{2}$ M. Müller $\odot,{ }^{3,4}$ \\ F. Schmidt-Kaler@ ${ }^{1}$, and U. G. Poschinger ${ }^{1, *}$ \\ ${ }^{1}$ Institut für Physik, Universität Mainz, Staudingerweg 7, 55128 Mainz, Germany \\ ${ }^{2}$ Departamento de Física Teórica, Universidad Complutense, Madrid 28040, Spain \\ ${ }^{3}$ Institute for Quantum Information, RWTH Aachen University, D-52056 Aachen, Germany \\ ${ }^{4}$ Peter Grünberg Institute, Theoretical Nanoelectronics, Forschungszentrum Jülich, D-52425 Jülich, German
}

(Received 16 July 2021; revised 30 November 2021; accepted 24 December 2021; published 17 February 2022)

\begin{abstract}
Quantum error correction requires the detection of errors via reliable measurements of multiqubit correlation operators. As these operations are inherently faulty, fault-tolerant schemes for realizing quantum error correction are required. Recently, a paradigm requiring only minimal resource overhead in the form of "flag" qubits to detect and correct errors has been proposed. We experimentally demonstrate a fault-tolerant weight-4 parity-check measurement scheme, where one additional flag qubit serves to detect errors, which would otherwise proliferate into uncorrectable weight- 2 errors onto the qubit register. We achieve a parity measurement fidelity of $92.3(2) \%$, which increases to $93.2(2) \%$ upon conditioning to the flag readout result, which shows that the measurement scheme intercepts intrinsic errors occurring throughout the sequence. We show that the protocol is capable of reliably intercepting faults by deliberately injecting bit- and phase-flip errors. For holistic benchmarking of the parity measurement scheme, we use an entanglement witnessing scheme requiring a minimal number of measurements to verify genuine six-qubit multipartite entanglement. The demonstrated fault-tolerant parity measurement scheme constitutes the key building block in a broad class of resource-efficient flag-based quantum error correction protocols including topological color codes. Our hardware platform is based on atomic ions stored in a microchip ion trap. The qubit register is dynamically reconfigured via shuttling operations enabling effective full connectivity without operational cross talk, thereby providing key prerequisites underlying fault-tolerant circuit design. These architectural features in combination with the demonstrated approach to flag-based fault-tolerant quantum error correction open up a route toward scalable fault-tolerant quantum computing.
\end{abstract}

\section{INTRODUCTION}

Quantum computers promise to outperform classical processors for particular tasks [1-4]. Solving problems beyond the reach of classical computers with a universal quantum computer requires the implementation of quantum error correction (QEC) protocols [5] to mitigate faulty operational building blocks. In QEC codes, logical qubits are encoded into entangled states of several physical qubits. Error syndrome readout permits detection of errors through quantum nondemolition parity-check measurements (PCMs) on the logical qubits [6-9]. Such a PCM requires performing a sequence of entangling gates between a set of data qubits and auxiliary qubits, to which the parity information is

*poschin@uni-mainz.de

Published by the American Physical Society under the terms of the Creative Commons Attribution 4.0 International license. Further distribution of this work must maintain attribution to the author(s) and the published article's title, journal citation, and DOI. mapped [10]. Projective measurements on the auxiliary qubits discretize eventual errors and thus allow for their detection and subsequent correction. However, PCM circuits inevitably consist of faulty gate operations and may therefore introduce additional errors during a QEC cycle, potentially corrupting the qubit register. Therefore, fault-tolerant (FT) QEC schemes are needed to prevent uncontrolled proliferation of errors [11]. Previously conceived FT PCM schemes required adding as many auxiliary qubits as the parity-check generator with maximum weight $[12,13]$. More recently proposed FT PCM schemes based on so-called flag qubits substantially reduce the overhead in terms of qubits and gate operations [14-19,19-21]. In particular, for distance-three codes implemented in fully connected quantum registers, a total of only two auxiliary qubits is sufficient to enable faulttolerant operation of the code [15], i.e., to guarantee the correctability of any single error occurring per QEC cycle, on any of the qubits or operations involved in the logical qubit.

To date, several QEC protocols and components have been demonstrated using trapped ions [22-28], superconducting 
circuits [29-32], nuclear magnetic resonance [33,34], or nitrogen-vacancy centers $[35,36]$. Increasing gate fidelities for different platforms [37-40] render QEC circuit noise thresholds [41] to be within reach of experimental capabilities. So far, with regard to FT QEC elements, FT state preparation and detection on primitives of topological surface codes have been realized with superconducting circuits $[9,29,42]$ or trapped ions [43]. Recently, FT preparation and FT operations of an encoded qubit on a distance-3 Bacon-Shor code was demonstrated [44], where the FT syndrome extraction was realized using four auxiliary qubits in addition to the nine data qubits.

In this work, we employ a trapped-ion quantum processor to demonstrate a flag-based weight-4 FT PCM scheme, which reduces the overhead for FT syndrome readout to two auxiliary qubits termed syndrome and flag. The flag qubit detects hook errors, i.e., faults occurring on the syndrome qubit that proliferate onto the data-qubit register. These remain undetectable in a non-FT PCM scheme and eventually result in a logical error.

\section{A. Stabilizer codes}

A weight-4 PCM circuit represents a key building block of the smallest distance-3 topological color code as well as for FT circuit constructions for larger 2D topological QEC codes $[15,19,45]$. The distance- 3 code depicted in Fig. 1 is equivalent to the $[[n=7, k=1, d=3]]$ Steane code [46,47]. This stabilizer code [48] encodes $k=1$ logical qubit into $n=7$ physical qubits and can therefore correct up to $t=(d-1) / 2=1$ arbitrary error on any of the physical qubits. This is guaranteed provided that QEC cycles are realized via fault-tolerant operations as we outline below, based, e.g., on the flag-qubit-based FT PCM measurement demonstrated in this work. The physical qubits comprising the code can be arranged in a $2 \mathrm{D}$ triangular lattice structure formed by three interconnected four-qubit plaquettes, as displayed in Fig. 1(a). The set of parity check or stabilizer generators $\left\{g_{i}\right\}$ of the code generates the stabilizer group $\mathcal{S}$ and consists of four-qubit Pauli operators defined on the vertices $v(p)$ of each its plaquettes $p$ :

$$
g_{x}^{(p)}=\underset{i \in v(p)}{\otimes} X_{i}, \quad g_{z}^{(p)}=\underset{i \in v(p)}{\bigotimes} Z_{i}
$$

with the Pauli matrices $X_{i}, Y_{i}, Z_{i}, \mathbb{1}_{i}$ pertaining to qubit $i$. The code space $\mathcal{L}$ hosting the logical qubit is fixed as the common two-dimensional eigenspace of eigenvalue +1 of all generators $g_{i}$ (and combinations thereof),

$$
\mathcal{L}:=\left\{|\psi\rangle_{\mathcal{L}}: g_{i}|\psi\rangle_{\mathcal{L}}=+|\psi\rangle_{\mathcal{L}} \forall g_{i}\right\}
$$

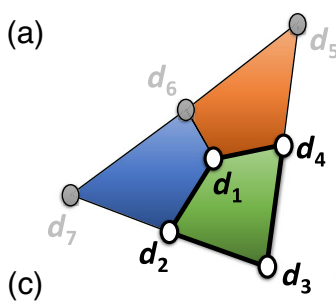

(b)

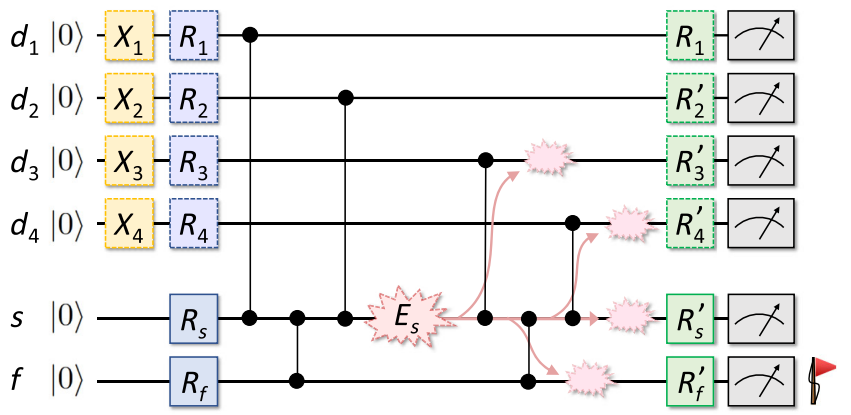

FIG. 1. (a) Sketch of the [[7, 1,3]] color code highlighting a plaquette comprised of data qubits $d_{1}-d_{4}$. (b) Segmented microchip ion trap showing how the qubits are distributed at the beginning of the PCM sequence. (c) Quantum circuit realizing the FT PCM on four data qubits. Two additional gates couple the syndrome to the flag qubit, allowing for detecting potentially uncorrectable faults. An example error $E_{s}$ (red) occurring throughout the third entangling gate results in a weight-2 error on the data-qubit register but is detected on the flag qubit. Initialization (yellow, blue) rotations are carried out at the beginning of the sequence; analysis rotations (green) are carried out before projective readout (gray).

\section{B. Fault-tolerant parity-check measurement}

A weight-4 PCM maps the parity of the data qubits belonging to one plaquette onto the syndrome qubit. For the case of a $Z$-type stabilizer, a series of consecutive phase gates is performed between each data qubit and the syndrome, which is initialized in $|-\rangle=(1 / \sqrt{2})(|0\rangle-|1\rangle)$. Each gate leads to a phase shift of $+\pi / 2$ or $-\pi / 2$, depending on the respective data qubit being in state $|0\rangle$ or in $|1\rangle$. This leads to the accumulation of a total phase shift given by the parity of the data qubits in the logical $Z$ basis. For uneven (even) parity, the syndrome qubit ends up in state $|-\rangle(|+\rangle)$, such that the outcome of a projective measurement on the syndrome qubit in the $X$ basis reveals the parity. For $X$-type stabilizers, the PCM requires an additional mapping of the data qubits from the $X$ basis to the $Z$ basis before the gate sequence. Faults can occur on any of the qubits during storage or can be caused by faulty entangling gates. Subsequent entangling gates lead to proliferation onto the participating qubits and therefore weight- 2 errors, as shown in Fig. 2.

Single-bit-flip errors $X_{i}$ on any data qubit $i$ will manifest as a -1 measurement outcome for each $Z$-type parity check on a plaquette containing qubit $i$. Likewise, a phase flip error $Z_{i}$ leads to a -1 measurement outcome for an $X$-type PCM. 


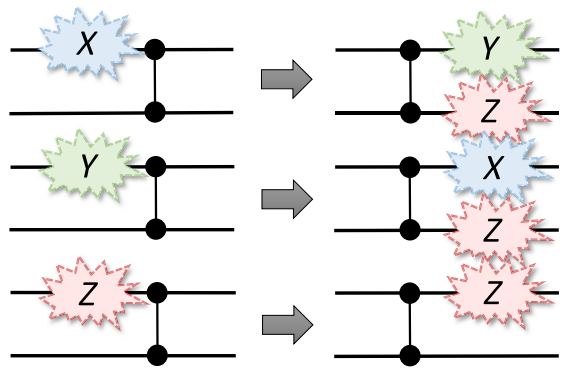

FIG. 2. Error propagation through $Z Z$ gates. The propagation of a Pauli- $X(Y)$ error leads to a conversion of the error into a $Y(X)$-type error, respectively, while a $Z$ error is triggered on the second qubit. A $Z$ error commutes with the $Z Z$ gate and thereby propagates through the gate without affecting the other qubit.

The classical information acquired from the PCMs is used to infer the error syndrome, i.e., the most likely error events that have affected the qubit register. To remove the conjectured error(s), suitable corrections are applied either by feedback onto the qubit register or as updates realized within the control software. Two simultaneous errors of the same type, e.g., a weight- 2 bit-flip error on qubits 3 and $4, E=X_{3} X_{4}$, are not correctable because they will result in the same error syndrome as a single-qubit-error event, $X_{5}$ in this example. In such a case, the subsequent error-correction attempt based on the obtained syndrome, here applying the "correction" $X_{5}$, would not remove the error, but rather result in a logical bit-flip error $X_{L}=X_{3} X_{4} X_{5}$ and thus a failure of the QEC cycle. This example shows the necessity of constructions and implementations of FT quantum circuits for parity-check measurements, i.e., in a way that single faults resulting during these circuits do not incur in undetected weight-2 errors on the code qubits.

A FT QEC cycle $[14,17]$ for the distance $d=3$ color code can be realized by a sequence of six PCMs using the flag-qubit-based circuit shown in Fig. 1. To prevent PCMinduced weight- 2 error events from going unnoticed, the syndrome qubit is coupled via two entangling gates to the second auxiliary qubit, which acts as a "flag": This coupling ensures that detrimental errors also propagate onto the flag qubit. Therefore, a -1 outcome of the flag qubit measurement signals the possible occurrence of a weight- 2 error propagation onto the data qubits. If a flag qubit is triggered in this first round of stabilizer generator measurements, this information can be combined with information from an additional second round of PCMs. This allows one to uniquely identify the occurrence of possible weight- 2 error events, distinguish them from other errors, e.g., single measurement errors, and thereby eventually correct them (see, e.g., Refs. [14,17] for a detailed discussion). This guarantees that any single error event occurring on any of the data or auxiliary qubits or the operations involved remains correctable, thereby establishing fault tolerance and completing one FT QEC cycle.
Here, we focus on the experimental verification of the fundamental building block of the flag-qubit QEC paradigm, namely, a flag-based FT weight 4 parity check $g_{z}=$ $Z_{1} Z_{2} Z_{3} Z_{4}$ according to the circuit shown in Fig. 1(c). The $g_{x}$ parity check is equivalent, as it merely requires mapping the data qubits by local rotations to the $X$ basis before syndrome readout. As we discuss, two additional entangling gates between the syndrome and flag qubits serve to catch error events throughout the PCM, which would otherwise result in weight- 2 errors on the data-qubit register [see Fig. 1(c)].

QEC is intimately linked to multipartite entanglement [49-53]. Several works reveal explicit connections between QEC and the generation of maximally entangled states and equivalently, between entanglement fidelities of the encoded states and the weight distribution of a code [54-57]. The inherent relation of nonclassical correlations as a prerequisite for QEC renders the generation and verification of genuine multipartite entanglement (GME) states to be a suitable and efficient benchmarking protocol for FT QEC building blocks. Here, we verify GME between the data and auxiliary qubits in order to demonstrate the correct functioning of our FT PCM and to benchmark the capabilities of our trapped-ion processor in the context of FT QEC.

\section{SHUTTLING-BASED TRAPPED-ION PLATFORM}

Quantum computer platforms based on trapped atomic ions arranged as static linear registers and laser addressing have seen substantial progress [58-60]. On such platforms, QEC building blocks have been demonstrated, such as repeated syndrome extraction and correction [27,61], encoding, readout and gate operations for the $[[7,1,3]]$ code [62], and entanglement of encoded logical qubits [63]. However, QEC protocols impose stringent demands on the scalability of the underlying hardware platform. The shuttling-based "quantum-CCD" approach offers a route to increased scalability [64-67]. Here, small subsets of the qubit ions are kept at distinct storage locations within a microstructured trap array, and the register is dynamically reconfigured via shuttling operations. This way, the excellent degree of control can be retained for increasing register sizes. In this work, we implement a shuttling-based FT PCM protocol. Between subsequent gate operations on two qubits, the register is reconfigured via shuttling operations. A special feature of our protocol is that we establish the required effective all-to-all connectivity by reordering the register via physical rotation of two commonly confined ions. This operation is equivalent to a unit-fidelity SWAP logic gate [68] and contrasts with faulty radiation-driven SWAP gates. This, together with the inherently low cross talk of the shuttling-based architecture, allows one to maintain the one-fault QEC condition. 
We employ a microstructured, segmented radio-frequency ion trap [66] consisting of 32 uniform segment pairs which are linearly arranged along a trap axis. Each segment pair can generate a confining potential well. The minimum distance between a trapped ion and a trap surface is about $160 \mu \mathrm{m}$, leading to axial and transverse heating rates in the regime of a few phonons per second. All qubit operations - initialization, gates, and readout - are carried out using laser beams, which are directed to segment 19 , henceforth referred to as the laser interaction zone (LIZ). Shuttling operations are carried out by supplying suitable voltage waveforms to the trap electrodes. Potential wells containing one or two qubits can be moved along the trap axis $[69,70]$, two commonly confined ions can be separated into potential wells [71,72], two separately confined ions can be merged into one well, and two commonly confined ions can be rotated such that their ordering along the trap axis is reversed [68]. The separate and merge, and swap shuttling operations are limited to the LIZ.

The qubits are encoded in the spin of the valence electron of atomic ${ }^{40} \mathrm{Ca}^{+}$ions $[73,74]$ with the assignment $|0\rangle \equiv\left|S_{1 / 2}, m_{J}=+1 / 2\right\rangle,|1\rangle \equiv\left|S_{1 / 2}, m_{J}=-1 / 2\right\rangle$. Local qubit rotations are carried out by driving a stimulated Raman transition with two beams near $397 \mathrm{~nm}$, with a frequency difference matching the qubit frequency. The bichromatic drive fields are detuned from the $S_{1 / 2} \leftrightarrow P_{1 / 2}$ transition by about $2 \pi \times 1 \mathrm{THz}$. Arbitrary local rotations on qubit $i$ of the form

$$
R_{i}(\theta, \phi)=\exp \left[-i \frac{\theta}{2}\left(\cos \phi X_{i}+\sin \phi Y_{i}\right)\right]
$$

can also be carried out simultaneously on two qubit ions commonly confined in the LIZ, in which case the Pauli operators are to be replaced by the respective tensor sum operators. The two drive beams are copropagating; therefore, the effective wave vector is zero, and the qubit drive does not couple to the motion of the qubit ions. Single-qubit Clifford error-per-gate rates of down to $2 \times 10^{-4}$ are measured via randomized benchmarking. Local qubit rotations occurring after initial rotations are corrected for systematic phases which are separately calibrated; see Sec. A 3.

Entangling gates between any two qubits $i$ and $j$ are realized via spin-dependent optical dipole forces [75] effecting a phase shift $\Phi$ between even- and odd-parity (with respect to the $Z$ basis) states represented by the unitary

$$
Z Z_{i j}(\Phi)=e^{(i / 2) \Phi Z_{i} \otimes Z_{j}} .
$$

The gates are driven by two laser beams at around $397 \mathrm{~nm}$ with a red detuning of about $2 \pi \times 1.0 \mathrm{THz}$ from the $S_{1 / 2} \leftrightarrow$ $P_{1 / 2}$ transition and are aligned such that the effective $\vec{k}$ vector is oriented perpendicular to the trap axis. The beams are arranged in a lin- $\perp$-lin polarization geometry; the beat pattern therefore has a polarization gradient and leads to a spin-dependent optical dipole force on the two ions. The frequency difference of the beams is tuned close to the transverse in-phase (gate) mode at $2 \pi \times 4.64 \mathrm{MHz}$, up to a detuning of $\delta \approx 2 \pi \times 20 \mathrm{kHz}$. This almost-resonant drive force leads to transient oscillatory excitation of the gate mode, returning to rest at a duration of $T=2 \pi / \delta \approx 50 \mu \mathrm{s}$. A geometric phase $\Phi$ proportional to the enclosed phase space area will be acquired, which can be tuned by the laser power. To realize a maximally entangling gate, a phase of $\Phi=\pi / 2$ is required.

The actual entangling gate operation consists of two gate pulses, each leading to a phase accumulation of $\Phi=\pi / 4$, interspersed by an additional rephasing pulse $R(\pi,-\pi / 2)$ with a typical duration of around $4 \mu \mathrm{s}$ after half the phase accumulation, which serves to maintain coherence [76]. This leads to a total gate unitary of

$$
G_{i j}=Z Z_{i j}(\pi / 4) R(\pi,-\pi / 2) Z Z_{i j}(\pi / 4) .
$$

The gate pulses feature a Tukey-type shape ensuring adiabatic switching of the gate interaction. The reduced bandwidth of the gate pulses leads to suppression of errors from off-resonant excitation of spectator motional modes [77]. Upon gate operations, the potential well in the LIZ features single-ion secular frequencies of $2 \pi \times\{1.49$, $3.88,4.64\} \mathrm{MHz}$, with the lowest frequency pertaining to the trap axis. Entangling gates are carried out using the transverse in-phase collective vibrational mode at $2 \pi \times$ $4.64 \mathrm{MHz}$ as the gate-mediating mode. The laser-beam geometry is chosen such that the gate operations are insensitive to the collective modes oscillating along the trap axis, which accumulate excitation from shuttling operations [78]. For a two-ion crystal, after ground-state cooling of all four transverse modes, typical two-qubit gate fidelities of around 99.6(2)\% are reached at a total gate duration of $120 \mu$ s verified via subspace cycle benchmarking [79].

\section{A. Shuttling-based parity measurement sequence}

The shuttling schedule realizing the FT PCM is constructed from the primitive operations described above, such that the total count of shuttling operations and the maximum spatial extent of the register is minimized, while additional constraints such as the minimum number of empty trap segments between two qubit sets are always fulfilled. Initially, the qubits are stored pairwise in order $\left\{d_{2}, d_{1}\right\}$, $\{s, f\}$, and $\left\{d_{3}, d_{4}\right\}$. The ion pairs are sequentially moved to the LIZ, where all four transverse modes are cooled close to the ground state via resolved-sideband cooling [73], and the qubits are initialized to $|0\rangle$ via optical pumping. Then, the data-qubit sets $\left\{d_{2}, d_{1}\right\},\left\{d_{3}, d_{4}\right\}$ are moved to the LIZ, where they are separated. Each data qubit is again moved into the LIZ, where optional $\pi$ flips allow for preparation of any desired logical basis state. A similar procedure is then 


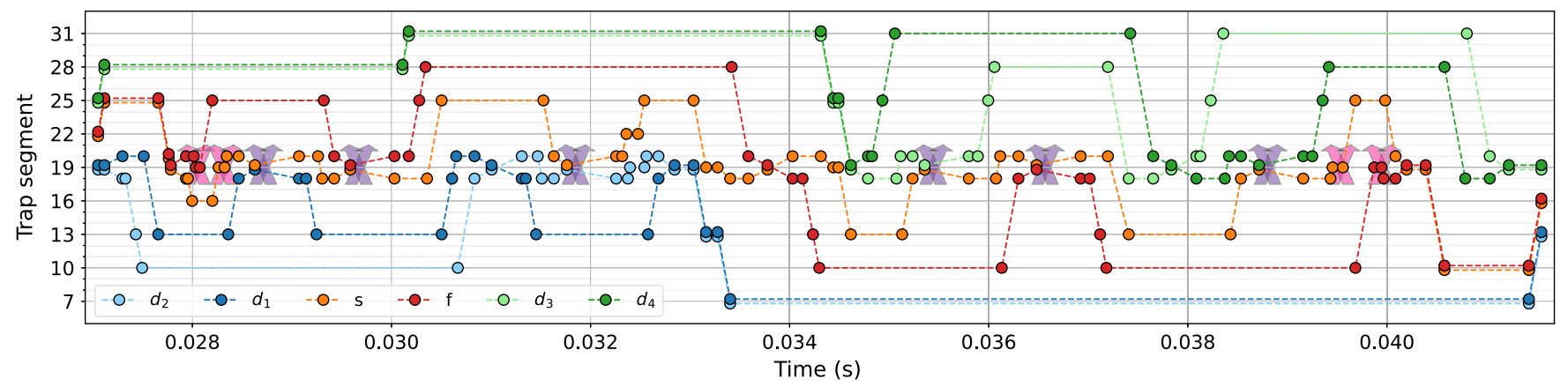

FIG. 3. Shuttling schedule of the fault-tolerant parity readout measurement sequence indicating how the ion qubits are moved between different storage sites of the segmented ion trap. The fixed laser interaction zone is located at segment 19. The arrows indicate laserdriven gate interactions. A distance of at minimum two empty segments between sets of ion qubits is maintained throughout the sequence. The maximum spatial extent of the register is 24 segments $(4.8 \mathrm{~mm})$.

carried out for the syndrome and flag qubits, which are prepared in superposition states via $\pi / 2$ rotations. Then, the parity-mapping sequence is carried out: The qubits undergo pairwise entangling gates according to Eq. (5) in the sequence $d_{1} s, s f, d_{2} s, d_{3} s, s f, d_{4} s$. Before and after each gate, an optional $\pi / 2$ rotation can be carried out on the participating data qubit in order to change the basis. Between two consecutive gates, a sequence of movement, separate and merge, and position-swap operations is carried out, bringing the qubit pair on which the following gate operation is to be carried out to the LIZ; see Fig. 3. Upon completion of the gate sequence, the syndrome and flag qubits are separately moved to the LIZ and each undergo an analysis $\pi / 2$ rotation. Qubit phases accumulated from positioning in the inhomogeneous magnetic field are calibrated via previous Ramsey-type measurements $[69,80]$ and are corrected for. Upon completion of the gate sequence, the qubits are kept pairwise in order $\left\{d_{2}, d_{1}\right\},\{s, f\}$, and $\left\{d_{3}, d_{4}\right\}$. These pairs are sequentially moved to the LIZ in reverse order, where laser-driven population transfer from $|0\rangle$ to the metastable $D_{5 / 2}$ state takes place. Then, the qubits are singled at the LIZ, where state-dependent laser-induced fluorescence is detected. Thresholding the number of detected photons allows for assigning detection events to logical $(Z)$ basis states and equivalently to eigenvalues $M_{i}= \pm 1$ of the Pauli operator $Z_{i}$ of qubit $i$ :

$$
\begin{gathered}
\text { "dark" } \rightarrow|0\rangle \Leftrightarrow M_{i}^{(Z)}=+1, \\
\text { "bright" } \rightarrow|1\rangle \Leftrightarrow M_{i}^{(Z)}=-1 .
\end{gathered}
$$

Logical results on rotated bases $M_{i}^{(X)}= \pm 1\left(M_{i}^{(Y)}= \pm 1\right)$ are acquired by performing an analysis rotation $R(\pi / 2,-\pi / 2)(R(\pi / 2,0))$ on the respective qubit before shelving and fluorescence detection. As the population transfer on all qubits is carried out before fluorescence detection, cross-talk errors throughout readout are avoided. Details on qubit and shuttling operations and the sequences for register preparation and readout can be found in the Appendix.

\section{MEASUREMENT RESULTS}

\section{A. Parity readout in the logical basis}

We first verify the functionality of the FT PCM protocol by carrying out the sequence shown in Fig. 3, while preparing the data qubits in all 16 computational basis states. The syndrome and flag qubit are initialized to $|-\rangle=$ $(1 / \sqrt{2})(|0\rangle-|1\rangle)$ by means of an $R(\pi / 2,-\pi / 2)$ rotation on the initial state $|0\rangle$. The measurement results $M_{s}^{(X)}$ of the syndrome are compared to the parity of the input state. We define the parity fidelity as

$$
\begin{aligned}
\mathcal{P}= & \frac{1}{2}\left[p\left(M_{s}^{(X)}=-1 \mid P_{\text {in }}=+1\right)\right. \\
& \left.+p\left(M_{s}^{(X)}=+1 \mid P_{\text {in }}=-1\right)\right],
\end{aligned}
$$

i.e., the probability of the correct syndrome readout result $M_{s}^{(X)}$ conditioned on the input parity $P_{\text {in }}$ of the data qubits. For 960 shots per input state, we measure $\mathcal{P}=92.3(2) \%$; see Fig. 4. For $93.7(2) \%$ of all shots, the flag qubit is detected as $M_{f}^{(X)}=-1$, indicating a low intrinsic rate of weight- 2 errors. Postselecting the syndrome measurement on the flag readout, i.e., taking only shots with flag result $M_{f}^{(X)}=-1$ into account, we obtain a conditional parity fidelity of $\mathcal{P}=93.2(2) \%$. It exceeds the bare parity fidelity by 4.5 standard errors, thus showing that the FT scheme operates in the regime where it can catch intrinsic weight-2 errors occurring throughout the PCM sequence. A discussion on the relevant error sources can be found in Sec. III C.

\section{B. Error injection}

In order to explicitly demonstrate that the FT PCM scheme can reliably detect hook errors, we deliberately 


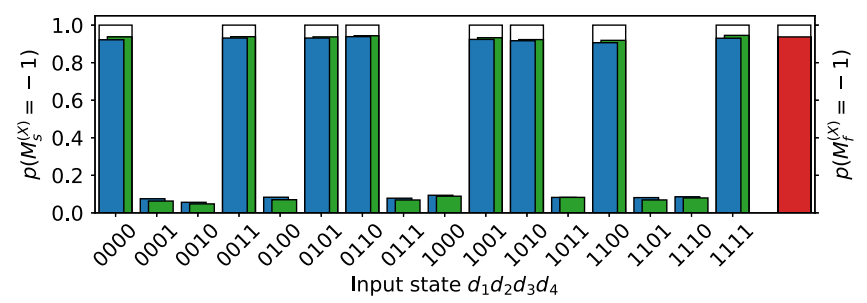

FIG. 4. Fault-tolerant parity readout. The syndrome $M_{s}^{(X)}=-1$ event rate is shown for each computational basis input state of the data qubits for all valid shots (blue) and postselected on the flag qubit (green), versus ideal rate (white). 960 shots per input state are measured; the average shot-noise error per input state is about $7 \times 10^{-3}$. The flag $M_{f}^{(X)}=-1$ readout rate is shown separated at the right (red).

inject errors $R_{s}(\pi, 0) \equiv X_{s}$ or $R_{s}(\pi, \pi / 2) \equiv Y_{s}$ on the syndrome qubit (equivalence up to a global phase) between gates $d_{2} s$ and $d_{3} s$; see Fig. 1 . The resulting $M_{s, f}^{(X)}=-1$ event rates for syndrome and flag are shown in Fig. 6. The injected $Y_{s}$, which corresponds to the simultaneous occurrence of a bit and a phase flip error, does not commute with the subsequent entangling gates Eq. (5). The three subsequent entangling gates involving the syndrome qubit lead to a final $X_{s}$ error, which is not detected upon syndrome measurement. It also proliferates to the data qubits in the form of $Z_{3}$ and $Z_{4}$ errors and would therefore compromise the encoded state, see Fig. 5. However, as it propagates to the flag as a $Z_{f}$ error via entangling gate $s f$, it can still be detected. We observe an error detection rate of $90.6(6) \% M_{f}^{(X)}=+1$ events on the flag. The syndrome still corresponds to the logical input state's parity with $\mathcal{P}=88.3(7) \%$. By contrast, the injected $X_{s}$ error results in a final $Y_{s}$ error, such that the final syndrome $M_{s}^{(X)}=-1$ events anticorrelate with the input parity, yielding $\mathcal{P}=14.7(7) \%$. Thus, as expected, the injected error results in a PCM measurement error. Similar to the $Y_{s}$ error, an error detection rate of $89.7(6) \% M_{f}^{(X)}=+1$ events is observed on the flag qubit. This indicates that the flag qubit again reliably detects the

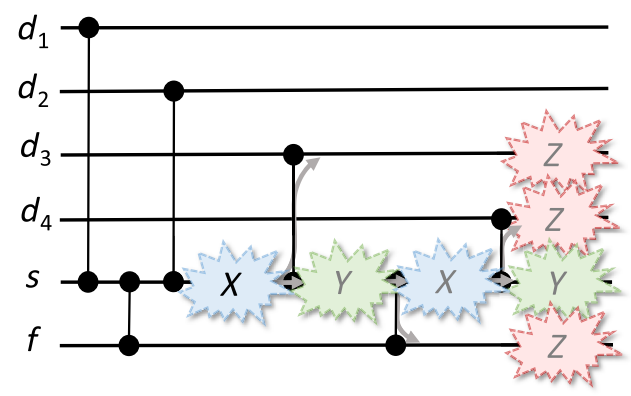

FIG. 5. Propagation of a Pauli- $X$ hook error within the FT PCM circuit inserted between gates $d_{2} s$ and $d_{3} s$. At each following $Z Z$ gate, the error on the syndrome itself is alternating between $X$ and $Y$, while a $Z$ error occurs on the other participating qubits.

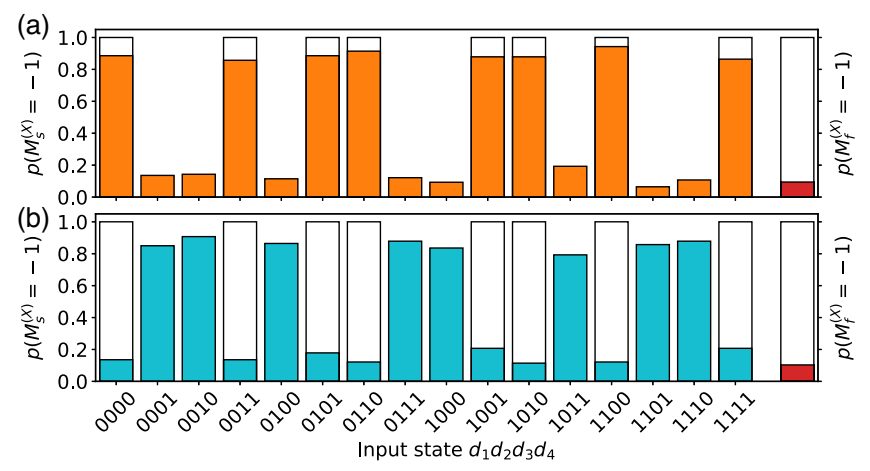

FIG. 6. Fault-tolerant parity readout including an injected error between gates $d_{2} s$ and $d_{3} s$. The syndrome $M_{s}^{(X)}=-1$ event rate is shown versus the logical input state of the data qubits. 140 shots per input state are measured, the average shot-noise error per input state is about $2.5 \times 10^{-2}$. (a) pertains to a $Y$-type error, which does not affect the syndrome readout. (b) pertains to an $X$-type error, which also flips the logical result of the syndrome. In both cases, the flag qubit is detected predominantly in $M_{f}^{(X)}=+1$ corresponding to detection of the error.

propagation of potentially detrimental weight- $2 Z$ errors onto the data qubits, as is required to preserve the fault tolerance of the scheme.

\section{Error analysis}

The achieved parity measurement fidelity is not yet sufficient for demonstration of above-threshold QEC; therefore, further measures for improving the operational fidelities are required. In this section, we discuss the possible error sources limiting the single-shot fidelity of the parity-check measurement. State preparation and measurement errors are of particular relevance in the context of QEC protocols: Many preparation and readout operations involving auxiliary qubits have to be performed per QEC cycle, and eventual feedback operations are conditioned on measurement results of these.

While the state preparation via two-stage optical pumping features an infidelity of $<0.1 \%$, which is dwarfed by measurement errors, the fidelity bottleneck here consists of the electron shelving operation for readout of the spin qubit (see Appendix B 3). The population transfer is accomplished using rapid adiabatic passage, which renders the process to be robust against modest coupling strength variations. The laser beam driving the population transfer is propagating perpendicularly to the axial (shuttling) direction, hence coupling mostly to weakly excited transverse motion. However, a presumed residual coupling to the strongly excited axial motion still leads to observable shuttlinginduced degradation of the readout efficiency. Readout errors are separately characterized by initializing all qubits in computational states $|0\rangle$ (dark readout) or $|1\rangle$ (bright readout) and performing the shuttling sequence without any further gates. Readout infidelities ranging between 1.2(2)\% and $1.8(3) \%$ are observed on the syndrome and flag qubits, 


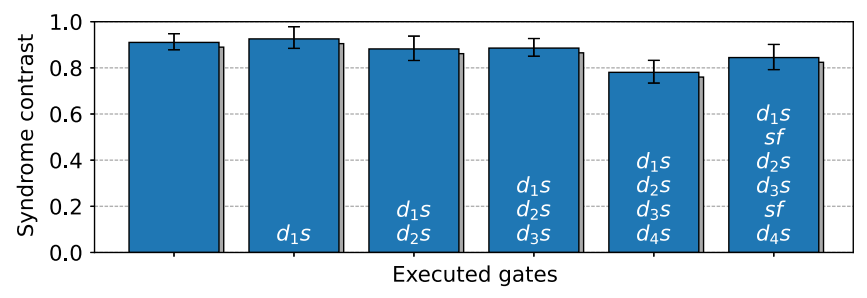

FIG. 7. Readout syndrome contrast after last gate with increasing number of gates executed, based on 160 shots per Pauli operator $X, Y$.

which undergo the most shuttling operations. For reference, a single-ion qubit without any shuttling operations before detection yields combined preparation and readout infidelities of $0.09(4) \%$ and $0.05(3) \%$ for $|0\rangle$ and $|1\rangle$, respectively.

Transverse secular motion accumulated from a shuttling operation may deteriorate subsequent two-qubit gates. The motion leads to dispersion of the coupling strength, which in turn leads to dispersion of the accumulated gate phase, finally manifesting as contrast loss on the syndrome and flag qubits. To characterize this effect, we measure the syndrome contrast for different gates toggled on or off, performing the complete shuttling sequence. The contrast is obtained from measuring expectation values $\left\langle X_{s}\right\rangle$ and $\left\langle Y_{s}\right\rangle$ and maximumlikelihood estimation. Contrast values ranging between 93 (5)\% and 78(5)\% are observed; see Fig. 7. A statistically significant dependence on the number of performed twoqubit gates is not supported by the data. We therefore conclude that this error source does not represent a relevant contribution to the infidelity of the parity-check measurement. This conclusion is further supported by thermometry on the transverse modes and a microscopic error model detailed in Appendix C 4, leading to estimated error contributions $\epsilon \ll 1 \%$.

The observed contrast loss on the syndrome without any two-qubit gate toggled on is 91(4)\%. This contrast loss can be attributed to fluctuations of the ambient magnetic field and is consistent with the observed parity measurement fidelity of 93.2(2)\%. Qubit dephasing from magnetic field fluctuations therefore constitutes the dominant error contribution.

\section{Timing budget and scalability}

The timing budget of the protocol is shown in Fig. 8 . About $23 \%$ of the duty cycle pertains to the PCM gate sequence, while the remainder pertains to register initialization-mostly cooling - and fluorescence readout. The time required for cooling the ion close to the ground state of the transverse modes could be substantially reduced by employing fast multimode cooling techniques such as electromagnetically induced transparency cooling [81] or polarization gradient cooling [82]. Of the actual PCM gate sequence, 5\% of the execution time is given by laser-driven gate interactions, while $95 \%$ is consumed by shuttling operation overhead.

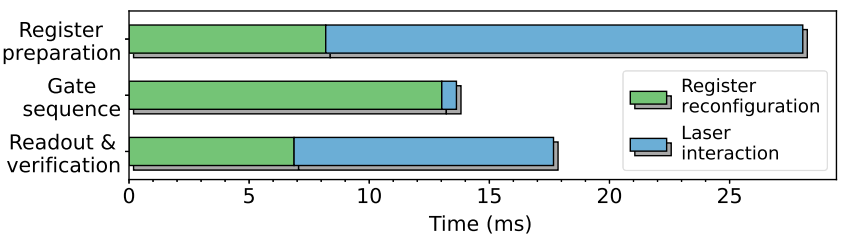

FIG. 8. Timing budget of the FT PCM sequence. The sequence is subdivided into register preparation, the actual PCM gate sequence shown in Fig. 3, and the readout and verification of all qubits. For each block, we show the timing overhead for shuttling operations (green) and laser-driven gate operations (blue).

While the preparation and readout overheads scale linearly with the register size, the shuttling overhead pertaining to the gate sequences can scale up quadratically with the qubit number, depending on the connectivity required by the underlying protocol. This overhead can be mitigated by improving the control hardware to decrease the time required for qubit register reconfigurations but also by using multiple manipulation sites for parallel processing [83]. We carry out a circuit of moderate depth without the requirement of in-sequence cooling for removing excitation of vibrational modes of the ions incurred from the shuttling operations. Future extensions will include dual-species operation for sympathetic cooling for a further increase of sequence depths and for nondestructive in-sequence readout $[84,85]$. In combination with the already achieved accuracy of two-qubit gate operations (see Sec. II) these technical improvements will pave the way for using the FT PCM demonstrated here as a building block for FT QEC cycles executed on complete logical qubits. As corroborated by a series of theory studies $[17,86,87]$, these efforts will eventually allow one to operate logical qubits in the regime of beneficial FT QEC “above threshold," in which logical qubits outperform their physical constituents.

\section{GENERATION OF GENUINE MULTIPARTITE ENTANGLEMENT}

The PCM scheme can be used to generate maximally entangled states. We verify four-qubit GME involving only the data qubits and extend it to the case of six-qubit GME, including the syndrome and flag qubits. The verification of $n$-qubit GME is carried out efficiently via measurement of witness operators [88,89], as the measurement overhead for complete state tomography [90] would scale unfavorably with $n$.

In contrast to the measurements discussed before, now all data qubits are initialized in $|+\rangle=(1 / \sqrt{2})(|0\rangle+|1\rangle)$ via local rotations $R_{d_{i}}(3 \pi / 2,-\pi / 2)$ applied to $|0\rangle$. The GME states generated by the PCM are listed in Table I (lines 1-3). The GME states are verified via entanglement witnessing [88,91]. An entanglement witness $W$ is an observable whose expectation value is by construction positive or equal to zero 
TABLE I. Properties of the entangled states generated by the PCM circuit for suitable input states. We distinguish the cases of the $n=4$ data qubits and all $n=6$ qubits involved in the respective GME state. The GME state vectors (before any measurements) are shown in line 1 . The corresponding substates are shown in lines 2 and 3 . The set of stabilizer generators with eigenvalues +1 which fix the four-qubit states $|\psi\rangle_{\text {out }}^{(4)}$ and $\left|\psi^{\perp}\right\rangle_{\text {out }}^{(4)}$, as well as the six-qubit state $|\psi\rangle_{\text {out }}^{(6)}$ are shown in line 4, witness operators $W_{n}$ constructed based on these generator sets according to Eq. (8) are displayed in line 5 together with the corresponding threshold values $l_{n}=(n-1) / n$ in line 6 .

\begin{tabular}{l|c|c|c}
\hline \hline & & $n=4$ & $n=6$ \\
\hline 1 & GME state $|\psi\rangle_{\text {out }}^{(n)}$ & $(1 / \sqrt{2})\left(|\psi\rangle_{\text {out }}^{(4)}|-\rangle_{s}+\left|\psi^{\perp}\right\rangle_{\text {out }}^{(4)}|+\rangle_{s}\right)$ & $(1 / \sqrt{2})\left(|\psi\rangle_{\text {out }}^{(5)}|-\rangle_{f}+i\left|\psi^{\perp}\right\rangle_{\text {out }}^{(5)}|+\rangle_{f}\right)$ \\
\hline 2 & Substate $M_{s / f}^{(X)}=+1$ & $|\psi\rangle_{\text {out }}^{(4)}=(1 / \sqrt{2})(|--++\rangle+|++--\rangle)$ & $|\psi\rangle_{\text {out }}^{(5)}=(1 / \sqrt{2})(|--++-\rangle+|++--+\rangle)$ \\
\hline 3 & Substate $M_{s / f}^{(X)}=-1$ & $\left|\psi^{\perp}\right\rangle_{\text {out }}^{(4)}=(1 / \sqrt{2})(|--++\rangle-|++--\rangle)$ & $\left|\psi^{\perp}\right\rangle_{\text {out }}^{(5)}=(1 / \sqrt{2})(|----+\rangle+|++++-\rangle)$ \\
\hline 4 & Stabilizer generator set $\mathcal{S}_{n}$ & $\left\{g_{1}=X_{1} X_{2}, g_{2}=-X_{2} X_{3}\right.$, & $\begin{array}{c}\left\{g_{1}=-X_{3} X_{s}, g_{2}=-X_{4} X_{s},\right. \\
g_{3} \\
=\end{array}$ \\
\hline 5 & Witness operator $\left.W_{n} X_{3} X_{4}, g_{4}= \pm Z_{1} Z_{2} Z_{3} Z_{4}\right\}$ & $\left.g_{1} Z_{2} Y_{f}, g_{6}=Z_{1} Z_{2} Z_{3} Z_{4} Z_{s}\right\}$ \\
\hline 6 & Bound $l_{n}$ & $l_{4} \mathbb{1}-\frac{1}{4} \sum_{i=1}^{4} g_{i}$ & $l_{6} \mathbb{1}-\frac{1}{6} \sum_{i=1}^{6} g_{i}$ \\
\hline \hline
\end{tabular}

for all separable states $\operatorname{tr}(W \rho) \geq 0$ and negative for specific entangled states $\operatorname{tr}(W \rho)<0$. The four- and six-qubit output states $|\psi\rangle_{\text {out }}^{(n)}$ of the PCM circuit belong to the class of stabilizer states, for which we use entanglement witness operators of the form

$$
W_{n}=l_{n} \mathbb{1}-\frac{1}{n} \sum_{i=1}^{n} g_{i}
$$

with the constant $l_{n}=(n-1) / n$. These witnesses correspond - up to a normalization factor $1 / n$ - to the witnesses proposed in Refs. [92,93]. They can be efficiently evaluated as they require the measurement of only the $n$ stabilizer generators $g_{i}$ (see Table I, line 4). The expected ideal output states can be uniquely defined as eigenstates of the stabilizer generators with eigenvalue +1 :

$$
g_{i}|\psi\rangle_{\text {out }}^{(n)}=+|\psi\rangle_{\text {out }}^{(n)} .
$$

Thus, GME in the experimentally prepared $n$-qubit states is signaled by a negative witness expectation value $\left\langle W_{n}\right\rangle<0$, which is the case if the sum of the generator expectation values $(1 / n) \sum_{i}^{n} g_{i}$ exceeds the threshold value of $l_{n}=(n-1) / n$, amounting to thresholds of $l_{4}=3 / 4$ and $l_{6}=5 / 6$ for the verification of four- and six-qubit GME, respectively. Each generator expectation value $\left\langle g_{i}\right\rangle$ is determined by measuring the qubit register in a measurement setting where each qubit $j$ is subjected to appropriate analysis pulses before readout, which feature drive phases corrected for systematic phases acquired throughout the PCM sequence.

For preparation of a four-qubit GME state, the entangling gates $s f$ between the syndrome and flag qubits are switched off; however, the respective rephasing pulses are retained.
The measured expectation values of the four stabilizer generators defined on the data qubits are shown in Fig. 9, each conditioned on the syndrome measurement result $M_{s}^{(X)}$. Consistent with the four-qubit GME state in Table I, $X_{1} X_{2}$, $X_{3} X_{4}\left(X_{2} X_{3}\right)$ display even (odd) parity, respectively. The parity of $Z_{1} Z_{2} Z_{3} Z_{4}$ depends conditionally on the syndrome readout result $M_{s}^{(X)}$, as upon syndrome readout, the four data qubits are projected into the respective \pm 1 eigenspace of the measured parity-check operator. For the input product state $|+\rangle^{\otimes 4}$ of the data qubits, they are projected into one of two four-qubit Greenberger-Horne-Zeilinger-type states. As the substates of the data qubits forming the GME state feature opposite parities in the $X$ basis, this shows that the PCM circuit reliably measures the parity in this basis upon initial basis change of the data qubits. Evaluating the entanglement witness expectation value conditionally on the syndrome measurement result, we obtain $\left\langle W_{4}\right\rangle=-0.14(1)$ for $M_{s}^{(X)}=+1 \quad$ and $\quad\left\langle W_{4}\right\rangle=-0.11(1) \quad$ for $\quad M_{s}^{(X)}=-1$,

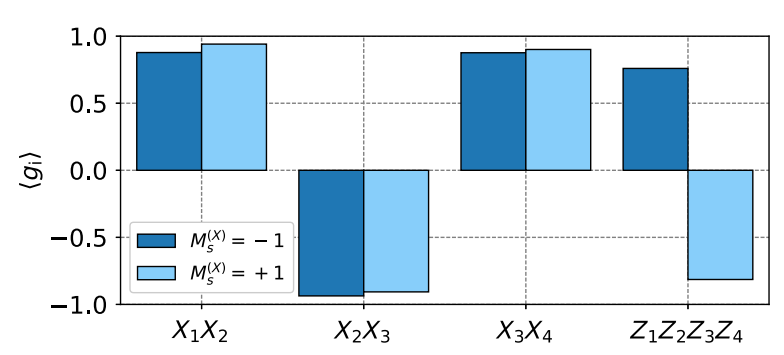

FIG. 9. Expectation values of stabilizer generators of the fourqubit GME state. About 330 per $X$-type stabilizer and 990 shots for the $Z$-type stabilizer are acquired. The results are conditioned on the readout result $M_{s}^{(X)}$ of the syndrome. The average shotnoise errors are about $2 \times 10^{-2}$ for all stabilizer expectation values. 


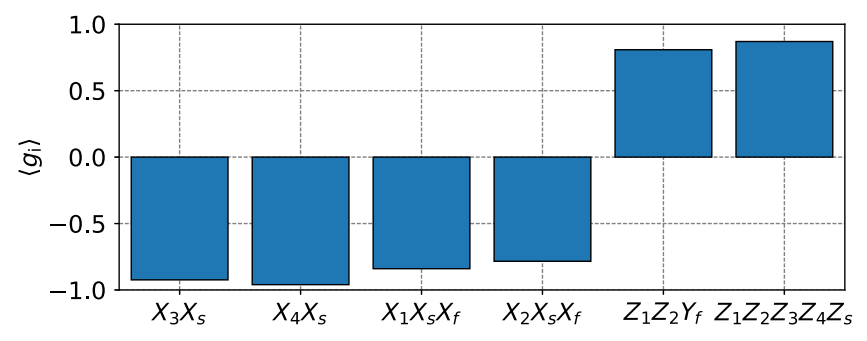

FIG. 10. Expectation values of stabilizer generators $g_{i}$ for verification of six-qubit GME. Each $X$-type stabilizer is evaluated from 500 shots, while the $Z$-type stabilizers are evaluated from 1000 shots each. The average shot-noise errors are about $2 \times 10^{-2}$ for all stabilizer expectation values.

respectively. Both values fall below zero by more than 10 standard errors; we therefore certify conditional fourqubit GME.

For the generation of six-qubit GME, we introduce additional rotations on the syndrome qubit $R_{s}(\pi / 2,0)$ between the $d_{2} s$ and $d_{3} s$ entangling gates [53] and $R_{s}(3 \pi / 2,-\pi / 2)$ directly before the analysis rotation. Note that the first of these rotations on the syndrome qubit can be interpreted in the QEC context as a coherent rotation error, which propagates through the subsequent gates and results in a six-qubit equal-weighted coherent superposition state. Here, the first component corresponds to the state where the error has propagated into two data-qubit errors captured by the flag qubit (in $|-\rangle_{f}$ ), and the second corresponds to the fault-free component (in $|+\rangle_{f}$ ). The measured stabilizer expectation values are shown in Fig. 10, from which we compute the expectation value of the witness $W_{6}$ (see Table I, line 5), obtaining $\left\langle W_{6}\right\rangle=-0.031(8)$. Falling below zero by 3.8 standard errors, we certify the capability of the flag-based PCM circuit to generate a six-qubit GME state.

\section{DISCUSSION AND OUTLOOK}

We successfully demonstrate a low-qubit-overhead FT PCM scheme on a shuttling-based trapped-ion quantum processor. To that end, we verify a parity measurement with high single-shot fidelity that increases when taking the flag qubit into account. By introducing errors deliberately, we show that the flag qubit reliably detects the occurrence of errors, which would otherwise proliferate into uncorrectable weight- 2 errors on the data-qubit register. This verifies the FT operation of the PCM as a key building block for QEC protocols. Furthermore, we efficiently and holistically benchmark the proper operation of the FT PCM scheme by witnessing four- and six-qubit GME generation for suitable input states. A key enabling feature of the FT PCM scheme is the virtually complete absence of cross-talk errors during gate operations. Negligible levels of cross talk are a key requirement and assumption, which underlies the very construction of FT quantum circuits. Beyond their relevance in the context of FT QEC, our results demonstrate the capability of realizing multiqubit quantum protocols with effective all-to-all connectivity on a shuttling-based quantum processor architecture.

\section{ACKNOWLEDGMENTS}

We acknowledge former contributions of Thomas Ruster, Henning Kaufmann, and Christian Schmiegelow to the experimental apparatus. The research is based upon work supported by the Office of the Director of National Intelligence (ODNI), Intelligence Advanced Research Projects Activity (IARPA), via the U.S. Army Research Office Grant No. W911NF-16-1-0070. The views and conclusions contained herein are those of the authors and should not be interpreted as necessarily representing the official policies or endorsements, either expressed or implied, of the ODNI, IARPA, or the U.S. Government. The U.S. Government is authorized to reproduce and distribute reprints for governmental purposes notwithstanding any copyright annotation thereon. Any opinions, findings, and conclusions or recommendations expressed in this material are those of the author(s) and do not necessarily reflect the view of the U.S. Army Research Office. We gratefully acknowledge funding from the EUH2020FETFLAG-2018-03 under Grant Agreement No. 820495, the European Research Council (ERC) via ERC StG QNets Grant No. 804247, and by the Germany Ministry of Science and Education via the VDI within the projects VERTICONS and IQuAn.

Note added.-Recently, we became aware of preprints containing work on related flag-qubit-based fault-tolerant QEC protocols $[94,95]$.

\section{APPENDIX A: QUBIT OPERATIONS}

\section{1. ${ }^{40} \mathrm{Ca}^{+}$spin qubit}

To store and process quantum information, the spin of the valence electron of ${ }^{40} \mathrm{Ca}^{+}$ions is used. The qubit is encoded in the Zeeman sublevels of the $S_{1 / 2}$ ground state, assigning $|0\rangle \equiv\left|S_{1 / 2}, m_{J}=+1 / 2\right\rangle \quad$ and $\quad|1\rangle \equiv\left|S_{1 / 2}, m_{J}=-1 / 2\right\rangle$ $[73,74]$. The relevant atomic transitions of ${ }^{40} \mathrm{Ca}^{+}$are shown in Fig. 11. Permanent magnets are used to produce a highly stable magnetic field of around $3.7 \mathrm{G}$, leading to a Zeeman splitting of around $2 \pi \times 10 \mathrm{MHz}$ between the qubit states. Ramsey coherence times of up to about $300 \mathrm{~ms}$ [74] are observed. The Zeeman splitting is smaller than the natural linewidth of the $S_{1 / 2} \leftrightarrow P_{1 / 2}$ transition, which facilitates Doppler cooling and detection.

\section{Initialization}

At the beginning of every measurement cycle, all ions are laser cooled (see Sec. B 2) and initialized to $|0\rangle \equiv\left|S_{1 / 2}, m_{J}=+1 / 2\right\rangle$. This initialization is performed 


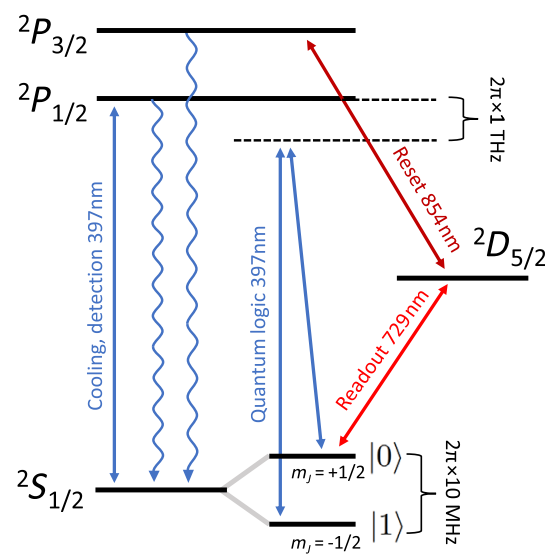

FIG. 11. Relevant atomic levels of a ${ }^{40} \mathrm{Ca}^{+}$ion encoding the spin qubit employed in the work.

by moving the three pairs of commonly confined ions sequentially into the LIZ and applying an optical pumping sequence. The sequence is a combination of two pumping stages. First, the qubits in the LIZ are exposed to a $\sigma_{+}$ polarized 397-nm beam for a duration of $1 \mu \mathrm{s}$, depleting the $|1\rangle$ state. To increase state preparation fidelity, a second, frequency-selective, pumping stage is employed. Here, four cycles of optical pumping are used, consisting of a $\pi$ pulse on the dipole-forbidden $\left|S_{1 / 2}, m_{J}=-1 / 2\right\rangle \leftrightarrow$ $\left|D_{5 / 2}, m_{J}=+3 / 2\right\rangle$ transition near $729 \mathrm{~nm}$, at a duration of about $10 \mu \mathrm{s}$. Each $\pi$ pulse is followed by exposure to the $854 \mathrm{~nm}$ "quench" laser for $4 \mu \mathrm{s}$, depleting the $D_{5 / 2}$ state again. This pumping scheme initializes all qubits to $|0\rangle$ with infidelity $<0.1 \%$.

In order to prepare different logical input states of the data qubits, the data bits are moved into the LIZ again after initialization to $|0\rangle$, where an optional rotation $R(\pi,-\pi / 2)$ is performed. This finalizes the register preparation part of the measurement sequence, which can be found in Fig. 12. The syndrome and flag are initialized to $|-\rangle$ by a single- qubit rotation $R(\pi / 2,-\pi / 2)$ applied at the beginning of the gate sequence.

Readout rotation pulses $R(\pi / 2,-\pi / 2)$ on the syndrome and flag are performed at the end of the gate sequence.

\section{Correction of systematic phases}

Throughout the dynamical reconfiguration of the qubits, ions are moved along the trap axis into different storage positions and acquire additional phases due to a small inhomogeneity of the magnetic field. The maximum difference of the qubit frequency is about $2 \pi \times 7 \mathrm{kHz}$ across the entire trap. Accumulated phases can be described by local $Z$ rotations, which commute with and therefore do not perturb the entangling gates. However, the analysis rotations ought to be corrected accordingly. We calibrate the phases from additional measurements, where the respective qubit is initialized in $|-\rangle$, and the shuttling sequence is carried out without executing entangling gates but with the rephasing pulses retained. Instead of the final analysis pulses, $X$ and $Y$ measurements are carried out. From the respective expectation values, we obtain the positioninginduced phases via maximum-likelihood estimation. With 40 shots per operator and qubit, we obtain a phase estimation accuracy of about $0.15 \mathrm{rad}$. For the measurement with injected errors, the phase $\phi_{\text {err }}$ of the error pulses on the syndrome are calibrated by scanning the phase of a local rotation $R\left(\pi, \phi_{\mathrm{err}}\right)$ at the error position to perform a full spin flip on the final syndrome readout. For the data qubits only, the analysis pulse phases are shifted by $-\pi / 2$ to take phase shifts from the entangling gates into account. For the GME generation and verification presented in Sec. IV, we apply the initial $3 \pi / 2$ rotation on each data qubit directly before the respective entangling gate to the syndrome and the analysis rotation directly after the gate. This way, the data qubits spend a minimum amount of time in a superposition state, which mitigates errors from dephasing.

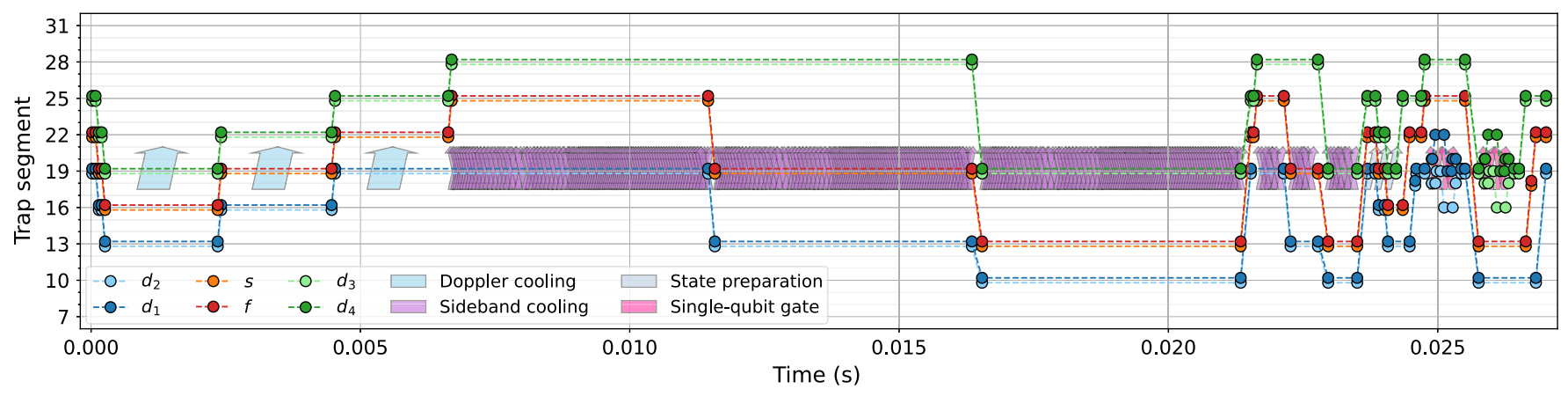

FIG. 12. Shuttling of the register preparation sequence part described in Sec. A 2. The two-qubit building blocks sequentially undergo Doppler laser cooling (light blue arrows, up to $6 \mathrm{~ms}$ ), pulsed sideband cooling on all transverse modes (purple arrows, up to $23 \mathrm{~ms}$ ), optical pumping (light blue arrows, at $24 \mathrm{~ms}$ ), and optional qubit rotations for preparation of arbitrary logical states of the data qubits (pink arrows, up to $26 \mathrm{~ms}$ ). 


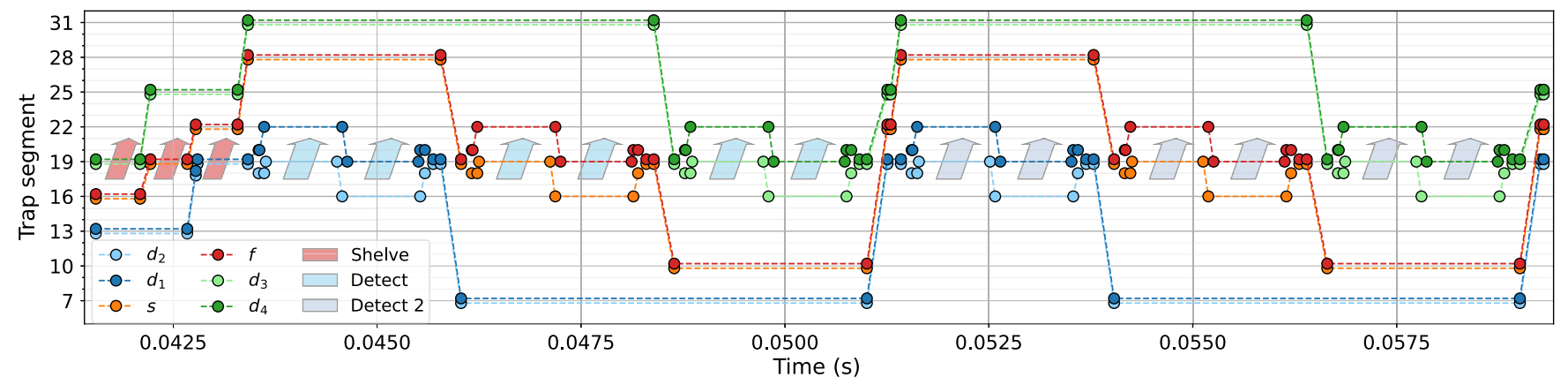

FIG. 13. Shuttling of the multiqubit readout sequence part described in Sec. B 3. The two-qubit building blocks sequentially undergo electron shelving (red arrows, up to $43 \mathrm{~ms}$ ). Then, state-dependent fluorescence is detected after separating each pair (light blue arrows, up to $51 \mathrm{~ms}$ ). Another round of detection is performed including qubit reset to verify register integrity (light blue arrows, up to $59 \mathrm{ms).}$

\section{APPENDIX B: REGISTER MANAGEMENT}

\section{Loading}

The six qubits are loaded in pairs of two commonly confined ion qubits. To that end, three potential wells are formed using the dc electrodes of the segmented trap. The wells are spaced by at least three empty segments to reduce the probability of unintentional additional loading events. ${ }^{40} \mathrm{Ca}^{+}$ions are obtained by resonant two-photon ionization from an effusive beam of ${ }^{40} \mathrm{Ca}$ atoms, using two laser beams near 374 and $423 \mathrm{~nm}$. The ions are usually trapped by applying a trapping voltage of $-2.4 \mathrm{~V}$ at the LIZ, while potential wells in storage regions use a trapping voltage of $-6 \mathrm{~V}$. Upon successful trapping, the ions are moved from the loading region to a storage position, and the next empty potential well is moved into the LIZ for loading. The potential wells are cycled through the LIZ until all qubits are stored at their desired location.

\section{Cooling}

In each measurement cycle, the ions are cooled close to the motional ground state via multiple cooling stages. First, Doppler cooling is performed using the $S_{1 / 2} \leftrightarrow$ $P_{1 / 2}$ transition near $397 \mathrm{~nm}$ and an exposure time of $2 \mathrm{~ms}$ per two commonly confined ions. The ion pairs are cooled in sequence $\left\{d_{3}, d_{4}\right\},\{s, f\}$, and $\left\{d_{2}, d_{1}\right\}$. The Doppler-cooled ions are further cooled by using pulsedresolved-sideband cooling via driving the stimulated Raman transition on the red sidebands of the corresponding transverse motional modes. Each cooling pulse realizes an approximate $\pi$ pulse on the transition

$$
|0\rangle|n\rangle \rightarrow|1\rangle|n-1\rangle,
$$

such that the phonon number $n$ of the driven secular mode is reduced. After each pulse, optical pumping using a circularly polarized laser near $397 \mathrm{~nm}$ at a pulse duration of $1 \mu$ s resets the state as

$$
|1\rangle|n-1\rangle \rightarrow|0\rangle|n-1\rangle .
$$

The ion pairs are sideband cooled sequentially in order $\left\{d_{2}, d_{1}\right\},\{s, f\}$, and $\left\{d_{3}, d_{4}\right\}$. First, all pairs undergo a cooling sequence of a total duration of $4 \mathrm{~ms}$, covering the second and first red sideband of all transverse modes for both axes perpendicular to the trap axis, in phase $2 \pi \times$ $\{3.88,4.64\} \mathrm{MHz}$ and out of phase $2 \pi \times\{3.57,4.37\}$ MHz. All ions which have already been cooled accumulate a small amount of excitation due to anomalous heating, mostly on the in-phase modes. This is mitigated by a second, much shorter, round of sideband cooling only on the in-phase modes, performed in the same cooling order. Mean thermal phonon numbers of $\lesssim 0.1$ are reached on all transverse modes of all two-ion blocks at the beginning of the sequence.

\section{Multiqubit readout}

The shuttling sequence for readout of all qubits is shown in Fig. 13. The sequence covers two rounds of detection for each qubit for reading out the state of the qubit and to ensure the validity of the measurement run. First, readout of the spin qubit requires electron shelving, i.e., selective population transfer from $|0\rangle$ to the metastable state $D_{5 / 2}$, which possesses a radiative lifetime of about $1 \mathrm{~s}$. This transfer is achieved by using rapid adiabatic passage pulses on the subtransitions $\left|0 \equiv S_{1 / 2}, m_{J}=+1 / 2\right\rangle \leftrightarrow\left|D_{5 / 2}, m_{J}=+1 / 2\right\rangle$ and $\left|0 \equiv S_{1 / 2}, m_{J}=+1 / 2\right\rangle \leftrightarrow\left|D_{5 / 2}, m_{J}=-3 / 2\right\rangle$. For both transitions, the ions are exposed to a chirped Gaussianshaped laser pulse varying the optical frequency by $\pm 60 \mathrm{kHz}$ around resonance within a duration of $200 \mu \mathrm{s}$. The pulse parameters are chosen to maximize the transfer probability from $|0\rangle$ and to minimize parasitic transfer from $|1\rangle$. The qubits undergo electron shelving pairwise in order $\left\{d_{3}, d_{4}\right\},\{s, f\}$, and $\left\{d_{2}, d_{1}\right\}$. After pairwise shelving of all ions, a nearly identical detection sequence is executed twice in reverse order. Here, all ion pairs are sequentially moved to the LIZ. Then, the pairs are separated, and the singled ions are consecutively moved to the LIZ. They are exposed to a laser beam resonantly driving the cycling transition near $397 \mathrm{~nm}$ at about onefold saturation. Scattered photons are 
collected and detected on a photomultiplier tube. Comparing the number of photons detected within $800 \mu$ s to a threshold allows for discrimination of $|0\rangle$ and $|1\rangle$. The same detection sequence is repeated, including an additional laser beam near $854 \mathrm{~nm}$, which depletes the metastable state via the $D_{5 / 2} \leftrightarrow$ $P_{3 / 2}$ electric dipole transition. A complete set of "bright" events at the second detection verifies that no ion losses have occurred during the measurement cycle. For the FT PCM shuttling sequence, we obtain a valid measurement cycle ratio of around $83 \%$. Finally, the ions are moved back to the initial loading positions.

\section{APPENDIX C: SHUTTLING OPERATIONS}

\section{Movement}

The microstructured, segmented radio-frequency ion trap consists of 32 pairs of dc electrodes referred to as trap segments. At most two ions are stored in one potential to harness the lower number of motional modes in a smaller ion crystal. In order to move ions to a neighboring segment, the voltage at the target segment is gradually put to negative bias, while the negative trapping voltage at the original segment is slowly increased at the same time. This way, the confining electrostatic potential well shifts from its original position to the destination segment. The voltage ramps are optimized to minimize the final motional excitation of the ions after the movement over a distance of one segment. The movement between two neighboring segments is performed within $20.9 \mu \mathrm{s}$. Transport over larger segment ranges is realized by concatenated application of singlesegment movements. A waiting time of $50.6 \mu$ s is inserted after the last shuttling operation before any laser-driven qubit operation is to be carried out. This ensures that the ions settle to the rest position in the LIZ.

\section{Separate and merge}

In order to obtain the required effective all-to-all connectivity within the six-qubit register, separation and merging of two qubit ions from or to common confinement is required. Separation is realized by dynamic control of the confining potential, transferring from a single-well to a double-well potential. In order to avoid excessive motional excitation from this operation, precise calibration of the process parameters is required. Most importantly, the rate of change of the harmonic confinement parameter at the transition point between axial confinement and anticonfinement needs to be sufficiently slow [71,72], which requires calibration of the trap potential via resolved-sideband spectroscopy. For this reason, the separate and merge process is currently limited to the LIZ segment. Furthermore, a "tilt" voltage difference between the neighboring segments of the LIZ is required to compensate stray fields along the trap axis, which needs to be calibrated to $1 \mathrm{mV}$ precision to ensure low residual motion after the separation. This voltage is automatically recalibrated throughout data acquisition, as its value may drift due to
UV-light-induced charge accumulation at the trap surfaces. A total separation duration of about $100 \mu \mathrm{s}$ is used. The merging process is merely the time-reverse operation employing the time-reverse voltage ramps with the same calibration parameters. The harmonic confinement along the trap axis is reduced throughout the separation and merge processes, down to a minimum value of about $2 \pi \times 220 \mathrm{kHz}$.

\section{Ion swap}

The swap operation is realized via physical rotation of two commonly confined ion qubits, i.e., flipping the positions of the two ions along the trap axis. The rotation is controlled using the dc trap electrodes comprising the LIZ and its neighboring segments. The two ions are confined within a pseudo-potential ellipsoid generated by the dc and ponderomotive trap potentials, where the principal axes define the secular frequencies. Before and after the rotation, these are $2 \pi \times\{1.49,3.88,4.64\} \mathrm{MHz}$ for the in-phase and $2 \pi \times\{2.57,3.57,4.37\} \mathrm{MHz}$ for the out-of-phase modes, where the lowest frequencies correspond to the axial (shuttling) direction. Throughout the swap process, the potential ellipsoid is rotated within the plane defined by the axial and lower-frequency transverse modes. We apply optimized voltage ramps computed from simulated trap potentials [96], which are designed to minimize the deviations of the secular frequencies throughout the rotation, given the available controls. We maintain a separation of at least $2 \pi \times 300 \mathrm{kHz}$ between the axial outof-phase and the lower-frequency transverse out-of-phase mode. This is of crucial importance: If transient near degeneracies or crossings of axial and transverse modes occur, transfer of motional excitation from the highly excited axial to the transverse modes takes places, which corrupts any subsequent entangling gate. All transverse modes are required to have low motional excitations $\lesssim 1$ phonon in order to realize high-fidelity entangling gates. The rotation process is carried out within a duration of $60 \mu \mathrm{s}$. The rotation-based swap does not require spectroscopic calibration and can therefore be performed at any trap segment [66]; however, this does not yield a reduction of the shuttling overhead, as the separation and merge processes are limited to the LIZ segment.

\section{Shuttling-induced gate errors}

In Sec. III C, we state that shuttling-induced excitation of the transverse modes does yield a significant contribution to the PCM error budget. Probing the motional excitation on the transverse modes, e.g., for qubit ions $d_{4}$ and $s$ at the position of the final two-qubit gate within the shuttling sequence, via sideband Rabi oscillation measurements $[66,72,97]$ yields mean phonon numbers $\bar{n} \lesssim 1$. We can therefore safely assume all transverse modes to be deeply within the Lamb-Dicke regime for all entangling gates within the shuttling sequence. 
Gate errors arising from an excited spectator mode can be accounted for by considering that for increasing quantum number $n$ of the respective mode, the ions are increasingly delocalized within the driving field [77]. This leads to a loss of coupling strength by a factor of $1-\eta^{2} n$, where $\eta$ is the Lamb-Dicke parameter. The gate error is then obtained by averaging over the phonon number distribution. It can be further assumed that the impact of excitation of the gate-mediating mode is quantitatively similar to a spectator mode. Extending the error model for the influence of four transverse modes, each characterized by a mean phonon number $\bar{n}_{i}$ and Lamb-Dicke parameter $\eta_{i}$, the error contribution can be estimated as

$\epsilon=\frac{\pi}{4} \sum_{\left\{n_{i}\right\}, i=1, \ldots, 4}\left(\prod_{i} p\left(n_{i}, \bar{n}_{i}\right)\right)\left(\prod_{i}\left(1-\eta_{i}^{2} n_{i}\right)-1\right)^{2}$.

The shuttling-induced excitation is of predominantly oscillatory nature; therefore, we insert a Poissonian distribution $p(n, \bar{n})=\exp (-\bar{n}) \bar{n}^{n} / n$ !. Assuming similar mean phonon numbers and Lamb-Dicke factors, we obtain

$$
\epsilon \approx \pi \eta^{4}\left(4 \bar{n}^{2}+\bar{n}\right) .
$$

Inserting $\eta=0.1$ and $\bar{n}=1.0$, the error contribution is merely $\epsilon \approx 0.15 \%$

[1] M. A. Nielsen and I. L. Chuang, Quantum Computation and Quantum Information (Cambridge University Press, Cambridge, England, 2000).

[2] A. Montanaro, Quantum Algorithms: An Overview, npj Quantum Inf. 2, 15023 (2016).

[3] F. Arute, K. Arya, R. Babbush, D. Bacon, J. C. Bardin, R. Barends et al., Quantum Supremacy Using a Programmable Superconducting Processor, Nature (London) 574, 505 (2019).

[4] Y. Alexeev, D. Bacon, K. R. Brown, R. Calderbank, L. D. Carr, F. T. Chong, B. DeMarco, D. Englund, E. Farhi, B. Fefferman et al., Quantum Computer Systems for Scientific Discovery, PRX Quantum 2, 017001 (2021).

[5] B. M. Terhal, Quantum Error Correction for Quantum Memories, Rev. Mod. Phys. 87, 307 (2015).

[6] D. B. Hume, T. Rosenband, and D. J. Wineland, HighFidelity Adaptive Qubit Detection through Repetitive Quantum Nondemolition Measurements, Phys. Rev. Lett. 99, 120502 (2007).

[7] A. Lupaşcu, S. Saito, T. Picot, P. C. de Groot, C. J. P. M. Harmans, and J. E. Mooij, Quantum Non-Demolition Measurement of a Superconducting Two-Level System, Nat. Phys. 3, 119 (2007).

[8] J. T. Barreiro, M. Müller, P. Schindler, D. Nigg, T. Monz, M. Chwalla, M. Hennrich, C. F. Roos, P. Zoller, and R. Blatt, An Open-System Quantum Simulator with Trapped Ions, Nature (London) 470, 486 (2011).
[9] A. D. Córcoles, E. Magesan, S. J. Srinivasan, A. W. Cross, M. Steffen, J. M. Gambetta, and J. M. Chow, Demonstration of a Quantum Error Detection Code Using a Square Lattice of Four Superconducting Qubits, Nat. Commun. 6, 6979 (2015).

[10] S. J. Devitt, W. J. Munro, and K. Nemoto, Quantum Error Correction for Beginners, Rep. Prog. Phys. 76, 076001 (2013).

[11] J. Preskill, in Introduction to Quantum Computation and Information (World Scientific Publishing Company, Singapore, 1996), p. 213.

[12] D. P. DiVincenzo and P. W. Shor, Fault-Tolerant Error Correction with Efficient Quantum Codes, Phys. Rev. Lett. 77, 3260 (1996).

[13] D. P. DiVincenzo and P. Aliferis, Effective Fault-Tolerant Quantum Computation with Slow Measurements, Phys. Rev. Lett. 98, 020501 (2007).

[14] R. Chao and B. W. Reichardt, Quantum Error Correction with Only Two Extra Qubits, Phys. Rev. Lett. 121, 050502 (2018).

[15] C. Chamberland and M. E. Beverland, Flag Fault-Tolerant Error Correction with Arbitrary Distance Codes, Quantum 2, 53 (2018).

[16] C. Chamberland and A.W. Cross, Fault-Tolerant Magic State Preparation with Flag Qubits, Quantum 3, 143 (2019).

[17] A. Bermudez, X. Xu, M. Gutiérrez, and S. C. Benjamin, and M. Müller, Fault-Tolerant Protection of Near-Term Trapped-Ion Topological Qubits under Realistic Noise Sources, Phys. Rev. A 100, 062307 (2019).

[18] B. W. Reichardt, Fault-Tolerant Quantum Error Correction for Steane's Seven-Qubit Color Code with Few or No Extra Qubits, Quantum Sci. Technol. 6, 015007 (2020).

[19] T. Tansuwannont, C. Chamberland, and D. Leung, Flag FaultTolerant Error Correction, Measurement, and Quantum Computation for Cyclic Calderbank-Shor-Steane Codes, Phys. Rev. A 101, 012342 (2020).

[20] C. Chamberland, A. Kubica, T. J. Yoder, and G. Zhu, Triangular Color Codes on Trivalent Graphs with Flag Qubits, New J. Phys. 22, 023019 (2020).

[21] R. Chao and B. W. Reichardt, Flag Fault-Tolerant Error Correction for Any Stabilizer Code, PRX Quantum 1, 010302 (2020).

[22] D. Nigg, M. Müller, E. A. Martinez, P. Schindler, M. Hennrich, T. Monz, M. A. Martin-Delgado, and R. Blatt, Quantum Computations on a Topologically Encoded Qubit, Science 345, 302 (2014).

[23] M. Müller, A. Rivas, E. A. Martínez, D. Nigg, P. Schindler, T. Monz, R. Blatt, and M. A. Martin-Delgado, Iterative Phase Optimization of Elementary Quantum Error Correcting Codes, Phys. Rev. X 6, 031030 (2016).

[24] P. Schindler, J. T. Barreiro, T. Monz, V. Nebendahl, D. Nigg, M. Chwalla, M. Hennrich, and R. Blatt, Experimental Repetitive Quantum Error Correction, Science 332, 1059 (2011).

[25] D. Kielpinski, A. Ben-Kish, J. Britton, V. Meyer, M. Rowe, W. Itano, D. Wineland, C. Sackett, and C. Monroe, Recent Results in Trapped-Ion Quantum Computing at NIST, Quantum Inf. Comput. 1, 113 (2001).

[26] J. Chiaverini, D. Leibfried, T. Schaetz, M. D. Barrett, R. B. Blakestad, J. Britton, W. M. Itano, J. D. Jost, E. Knill, 
C. Langer, R. Ozeri, and D. J. Wineland, Realization of Quantum Error Correction, Nature (London) 432, 602 (2004).

[27] V. Negnevitsky, M. Marinelli, K. K. Mehta, H.-Y. Lo, C. Flühmann, and J. P. Home, Repeated Multi-Qubit Readout and Feedback with a Mixed-Species Trapped-Ion Register, Nature (London) 563, 527 (2018).

[28] R. Stricker, D. Vodola, A. Erhard, L. Postler, M. Meth, M. Ringbauer, P. Schindler, T. Monz, M. Müller, and R. Blatt, Experimental Deterministic Correction of Qubit Loss, Nature (London) 585, 207 (2020).

[29] J. Kelly et al., State Preservation by Repetitive Error Detection in a Superconducting Quantum Circuit, Nature (London) 519, 66 (2015).

[30] N. Ofek, A. Petrenko, R. Heeres, P. Reinhold, Z. Leghtas, B. Vlastakis, Y. Liu, L. Frunzio, S. Girvin, L. Jiang, M. Mirrahimi, M. Devoret, and R. Schoelkopf, Extending the Lifetime of a Quantum Bit with Error Correction in Superconducting Circuits, Nature (London) 536, 441 (2016).

[31] C. K. Andersen, A. Remm, S. Lazar, S. Krinner, N. Lacroix, G. J. Norris, M. Gabureac, C. Eichler, and A. Wallraff, Repeated Quantum Error Detection in a Surface Code, Nat. Phys. 16, 875 (2020).

[32] Z. Chen, K. J. Satzinger, J. Atalaya, A. N. Korotkov, A. Dunsworth, D. Sank et al., Exponential Suppression of Bit or Phase Flip Errors with Repetitive Error Correction, arXiv:2102.06132.

[33] J. Zhang, R. Laflamme, and D. Suter, Experimental Implementation of Encoded Logical Qubit Operations in a Perfect Quantum Error Correcting Code, Phys. Rev. Lett. 109, 100503 (2012).

[34] E. Knill, R. Laflamme, and R. Martinez, and C. Negrevergne, Benchmarking Quantum Computers: The Five-Qubit Error Correction Code, Phys. Rev. Lett. 86, 5811 (2001).

[35] G. Waldherr, Y. Wang, S. Zaiser, M. Jamali, T. Schulte-Herbrüggen, H. Abe, T. Ohshima, J. Isoya, J. F. $\mathrm{Du}, \mathrm{P}$. Neumann et al., Quantum Error Correction in a Solid-State Hybrid Spin Register, Nature (London) 506, 204 (2014).

[36] T. Unden, P. Balasubramanian, D. Louzon, Y. Vinkler, M. B. Plenio, M. Markham, D. Twitchen, A. Stacey, I. Lovchinsky, A. O. Sushkov, M. D. Lukin, A. Retzker, B. Naydenov, L. P. McGuinness, and F. Jelezko, Quantum Metrology Enhanced by Repetitive Quantum Error Correction, Phys. Rev. Lett. 116, 230502 (2016).

[37] R. Srinivas, S. C. Burd, H. M. Knaack, R. T. Sutherland, A. Kwiatkowski, S. Glancy, E. Knill, D. J. Wineland, D. Leibfried, A. C. Wilson, D. T. C. Allcock, and D. H. Slichter, High-Fidelity Laser-Free Universal Control of Two Trapped Ion Qubits, Nature (London) 597, 209 (2021).

[38] C. R. Clark, H. N. Tinkey, B. C. Sawyer, A. M. Meier, K. A. Burkhardt, C. M. Seck, C. M. Shappert, N. D. Guise, C. E. Volin, S. D. Fallek, H. T. Hayden, W. G. Rellergert, and K. R. Brown, High-Fidelity Bell-State Preparation with ${ }^{40} \mathrm{Ca}^{+}$Optical Qubits, Phys. Rev. Lett. 127, 130505 (2021).

[39] M. Kjaergaard, M. E. Schwartz, A. Greene, G. O. Samach, A. Bengtsson, M. O Keeffe et al., Programming a Quantum Computer with Quantum Instructions, arXiv:2001.08838 [Phys. Rev. X (to be published)].
[40] A. Browaeys and T. Lahaye, Many-Body Physics with Individually Controlled Rydberg Atoms, Nat. Phys. 16, 132 (2020).

[41] R. Raussendorf and J. Harrington, Fault-Tolerant Quantum Computation with High Threshold in Two Dimensions, Phys. Rev. Lett. 98, 190504 (2007).

[42] M. Takita, A. W. Cross, A. D. Córcoles, and J. M. Chow, and J. M. Gambetta, Experimental Demonstration of FaultTolerant State Preparation with Superconducting Qubits, Phys. Rev. Lett. 119, 180501 (2017).

[43] N. M. Linke, M. Gutierrez, K. A. Landsman, C. Figgatt, S. Debnath, K. R. Brown, and C. Monroe, Fault-Tolerant Quantum Error Detection, Sci. Adv. 3, e1701074 (2017).

[44] L. Egan, D. M. Debroy, C. Noel, A. Risinger, D. Zhu, D. Biswas, M. Newman, M. Li, K. R. Brown, M. Cetina, and C. Monroe, Fault-Tolerant Control of an Error-Corrected Qubit, Nature (London) 598, 281 (2021).

[45] L. Lao and C. G. Almudever, Fault-Tolerant Quantum Error Correction on Near-Term Quantum Processors Using Flag and Bridge Qubits, Phys. Rev. A 101, 032333 (2020).

[46] A. M. Steane, Error Correcting Codes in Quantum Theory, Phys. Rev. Lett. 77, 793 (1996).

[47] H. Bombin and M. A. Martin-Delgado, Topological Quantum Distillation, Phys. Rev. Lett. 97, 180501 (2006).

[48] D. Gottesman, Stabilizer Codes and Quantum Error Correction, Ph.D. thesis, Caltech, 1997, arXiv:9705052.

[49] J. Preskill, Lecture Notes for Physics 219: Quantum Computation, California Institute of Technology, 1998.

[50] C. H. Bennett, D. P. DiVincenzo, J. A. Smolin, and W. K. Wootters, Mixed-State Entanglement and Quantum Error Correction, Phys. Rev. A 54, 3824 (1996).

[51] D. A. Lidar and T. A. Brun, Quantum Error Correction (Cambridge University Press, Cambridge, England, 2013).

[52] A. Almheiri, X. Dong, and D. Harlow, Bulk Locality and Quantum Error Correction in AdS/CFT, J. High Energy Phys. 04 (2015) 163.

[53] A. Rodriguez-Blanco, A. Bermudez, M. Müller, and F. Shahandeh, Efficient and Robust Certification of Genuine Multipartite Entanglement in Noisy Quantum Error Correction Circuits, PRX Quantum 2, 020304 (2021).

[54] A. J. Scott, Multipartite Entanglement, Quantum-ErrorCorrecting Codes, and Entangling Power of Quantum Evolutions, Phys. Rev. A 69, 052330 (2004).

[55] Z. Raissi, C. Gogolin, A. Riera, and A. Acín, Optimal Quantum Error Correcting Codes from Absolutely Maximally Entangled States, J. Phys. A 51, 075301 (2018).

[56] P. Shor and R. Laflamme, Quantum Analog of the MacWilliams Identities for Classical Coding Theory, Phys. Rev. Lett. 78, 1600 (1997).

[57] E. Rains, Quantum Weight Enumerators, IEEE Trans. Inf. Theory 44, 1388 (1998).

[58] T. Monz, P. Schindler, J. T. Barreiro, M. Chwalla, D. Nigg, W. A. Coish, M. Harlander, W. Hänsel, M. Hennrich, and R. Blatt, 14-Qubit Entanglement: Creation and Coherence, Phys. Rev. Lett. 106, 130506 (2011).

[59] S. Debnath, N. M. Linke, C. Figgatt, K. A. Landsman, K. Wright, and C. Monroe, Demonstration of a Small Programmable Quantum Computer with Atomic Qubits, Nature (London) 536, 63 (2016). 
[60] N. M. Linke, D. Maslov, M. Roetteler, S. Debnath, C. Figgatt, K. A. Landsman, K. Wright, and C. Monroe, Experimental Comparison of Two Quantum Computing Architectures, Proc. Natl. Acad. Sci. U.S.A. 114, 3305 (2017).

[61] P. Schindler, J. T. Barreiro, T. Monz, V. Nebendahl, D. Nigg, M. Chwalla, M. Hennrich, and R. Blatt, Experimental Repetitive Quantum Error Correction, Science 332, 1059 (2011).

[62] D. Nigg, M. Müller, E. A. Martinez, P. Schindler, M. Hennrich, T. Monz, M. A. Martin-Delgado, and R. Blatt, Quantum Computations on a Topologically Encoded Qubit, Science 345, 302 (2014).

[63] A. Erhard, H. P. Nautrup, M. Meth, L. Postler, R. Stricker, M. Stadler, V. Negnevitsky, M. Ringbauer, P. Schindler, H. J. Briegel, R. Blatt, N. Friis, and T. Monz, Entangling Logical Qubits with Lattice Surgery, Nature (London) $\mathbf{5 8 9}$, 220 (2021).

[64] D. Kielpinski, C. Monroe, and D. J. Wineland, Architecture for a Large-Scale Ion-Trap Quantum Computer, Nature (London) 417, 709 (2002).

[65] B. Lekitsch, S. Weidt, A. G. Fowler, K. Mølmer, S. J. Devitt, C. Wunderlich, and W. K. Hensinger, Blueprint for a Microwave Trapped Ion Quantum Computer, Sci. Adv. 3, e1601540 (2017).

[66] V. Kaushal, B. Lekitsch, A. Stahl, J. Hilder, D. Pijn, C. Schmiegelow, A. Bermudez, M. Müller, F. Schmidt-Kaler, and U. Poschinger, Shuttling-Based Trapped-Ion Quantum Information Processing, AVS Quantum Sci. 2, 014101 (2020).

[67] J. M. Pino, J. M. Dreiling, C. Figgatt, J. P. Gaebler, S. A. Moses, M. S. Allman, C. H. Baldwin, M. Foss-Feig, D. Hayes, K. Mayer, C. Ryan-Anderson, and B. Neyenhuis, Demonstration of the Trapped-Ion Quantum CCD Computer Architecture, Nature (London) 592, 209 (2021).

[68] H. Kaufmann, T. Ruster, C. T. Schmiegelow, M. A. Luda, V. Kaushal, J. Schulz, D. von Lindenfels, F. Schmidt-Kaler, and U. G. Poschinger, Fast Ion Swapping for QuantumInformation Processing, Phys. Rev. A 95, 052319 (2017).

[69] A. Walther, F. Ziesel, T. Ruster, S. T. Dawkins, K. Ott, M. Hettrich, K. Singer, F. Schmidt-Kaler, and U. G. Poschinger, Controlling Fast Transport of Cold Trapped Ions, Phys. Rev. Lett. 109, 080501 (2012).

[70] R. Bowler, J. Gaebler, Y. Lin, T. R. Tan, D. Hanneke, J. D. Jost, J. P. Home, D. Leibfried, and D. J. Wineland, Coherent Diabatic Ion Transport and Separation in a Multizone Trap Array, Phys. Rev. Lett. 109, 080502 (2012).

[71] T. Ruster, C. Warschburger, H. Kaufmann, C. T. Schmiegelow, A. Walther, M. Hettrich, A. Pfister, V. Kaushal, and F. Schmidt-Kaler, and U. G. Poschinger, Experimental Realization of Fast Ion Separation in Segmented Paul Traps, Phys. Rev. A 90, 033410 (2014).

[72] H. Kaufmann, T. Ruster, C. T. Schmiegelow, F. SchmidtKaler, and U. G. Poschinger, Dynamics and Control of Fast Ion Crystal Splitting in Segmented Paul Traps, New J. Phys. 16, 073012 (2014).

[73] U. G. Poschinger, G. Huber, F. Ziesel, M. Deiß, M. Hettrich, S. A. Schulz, K. Singer, G. Poulsen, M. Drewsen, R. J. Hendricks, and F. Schmidt-Kaler, Coherent Manipulation of a ${ }^{40} \mathrm{Ca}^{+}$Spin Qubit in a Micro Ion Trap, J. Phys. B 42, 154013 (2009).

[74] T. Ruster, C. T. Schmiegelow, H. Kaufmann, C. Warschburger, F. Schmidt-Kaler, and U. G. Poschinger, A Long-Lived Zeeman Trapped-Ion Qubit, Appl. Phys. B 122, 254 (2016).

[75] D. Leibfried, B. DeMarco, V. Meyer, D. Lucas, M. Barrett, J. Britton, W. M. Itano, B. Jelenkovic, C. Langer, T. Rosenband, and D. J. Wineland, Experimental Demonstration of a Robust, High-Fidelity Geometric Two Ion-Qubit Phase Gate, Nature (London) 422, 412 (2003).

[76] M. J. Biercuk, H. Uys, A. P. VanDevender, N. Shiga, W. M. Itano, and J. J. Bollinger, Optimized Dynamical Decoupling in a Model Quantum Memory, Nature (London) 458, 996 (2009).

[77] C. J. Ballance, High-Fidelity Quantum Logic in $\mathrm{Ca}^{+}$, University of Oxford, 2014.

[78] H. Kaufmann, T. Ruster, C. T. Schmiegelow, M. A. Luda, V. Kaushal, J. Schulz, D. von Lindenfels, F. Schmidt-Kaler, and U. G. Poschinger, Scalable Creation of Long-Lived Multipartite Entanglement, Phys. Rev. Lett. 119, 150503 (2017).

[79] C. H. Baldwin, B. J. Bjork, J. P. Gaebler, D. Hayes, and D. Stack, Subspace Benchmarking High-Fidelity Entangling Operations with Trapped Ions, Phys. Rev. Research 2, 013317 (2020).

[80] T. Ruster, H. Kaufmann, M. A. Luda, V. Kaushal, C. T. Schmiegelow, F. Schmidt-Kaler, and U. G. Poschinger, Entanglement-Based dc Magnetometry with Separated Ions, Phys. Rev. X 7, 031050 (2017).

[81] Y. Lin, J. P. Gaebler, T. R. Tan, R. Bowler, J. D. Jost, D. Leibfried, and D. J. Wineland, Sympathetic Electromagnetically-Induced-Transparency Laser Cooling of Motional Modes in an Ion Chain, Phys. Rev. Lett. 110, 153002 (2013).

[82] M. K. Joshi, A. Fabre, C. Maier, T. Brydges, D. Kiesenhofer, H. Hainzer, R. Blatt, and C. F. Roos, Polarization-Gradient Cooling of $1 D$ and $2 D$ Ion Coulomb Crystals, New J. Phys. 22, 103013 (2020).

[83] K. K. Mehta, C. Zhang, M. Malinowski, T.-L. Nguyen, M. Stadler, and J.P. Home, Integrated Optical Multi-Ion Quantum Logic, Nature (London) 586, 533 (2020).

[84] C. J. Ballance, V. M. Schäfer, J. P. Home, D. J. Szwer, S. C. Webster, D. T. C. Allcock, N. M. Linke, T. P. Harty, D. P. L. A. Craik, D. N. Stacey, A. M. Steane, and D. M. Lucas, Hybrid Quantum Logic and a Test of Bell's Inequality Using Two Different Atomic Isotopes, Nature (London) 528, 384 (2015).

[85] T. R. Tan, J. P. Gaebler, Y. Lin, Y. Wan, R. Bowler, D. Leibfried, and D. J. Wineland, Multi-Element Logic Gates for Trapped-Ion Qubits, Nature (London) 528, 380 (2015).

[86] A. Bermudez, X. Xu, R. Nigmatullin, J. O'Gorman, V. Negnevitsky, P. Schindler, T. Monz, U. G. Poschinger, C. Hempel, J. Home, F. Schmidt-Kaler, M. Biercuk, R. Blatt, S. Benjamin, and M. Müller, Assessing the Progress of Trapped-Ion Processors Towards Fault-Tolerant Quantum Computation, Phys. Rev. X 7, 041061 (2017).

[87] P. Parrado-Rodriguez, C. Ryan-Anderson, A. Bermudez, and M. Müller, Crosstalk Suppression for Fault-Tolerant Quantum Error Correction with Trapped Ions, Quantum 5, 487 (2021). 
[88] O. Guehne and G. Toth, Entanglement Detection, Phys. Rep. 474, 1 (2009).

[89] M. Bourennane, M. Eibl, C. Kurtsiefer, S. Gaertner, H. Weinfurter, O. Gühne, P. Hyllus, D. Bruß, M. Lewenstein, and A. Sanpera, Experimental Detection of Multipartite Entanglement Using Witness Operators, Phys. Rev. Lett. 92, 087902 (2004).

[90] C. Roos, M. Riebe, H. Häffner, W. Hänsel, J. Benhelm, G. Lancaster, C. Becher, F. Schmidt-Kaler, and R. Blatt, Control and Measurement of Three-Qubit Entangled States, Science 304, 1478 (2004).

[91] N. Friis, G. Vitagliano, M. Malik, and M. Huber, Entanglement Certification from Theory to Experiment, Nat. Rev. Phys. 1, 72 (2019).

[92] G. Tóth and O. Gühne, Entanglement Detection in the Stabilizer Formalism, Phys. Rev. A 72, 022340 (2005).

[93] G. Tóth and O. Gühne, Detecting Genuine Multipartite Entanglement with Two Local Measurements, Phys. Rev. Lett. 94, 060501 (2005).
[94] C. Ryan-Anderson, J. G. Bohnet, K. Lee, D. Gresh, A. Hankin, J. P. Gaebler, D. Francois, A. Chernoguzov, D. Lucchetti, N. C. Brown, T. M. Gatterman, S. K. Halit, K. Gilmore, J. Gerber, B. Neyenhuis, D. Hayes, and R. P. Stutz, Realization of Real-Time Fault-Tolerant Quantum Error Correction, Phys. Rev. X 11, 041058 (2021).

[95] M. H. Abobeih, Y. Wang, J. Randall, S. J. H. Loenen, C. E. Bradley, M. Markham, D. J. Twitchen, B. M. Terhal, and T. H. Taminiau, Fault-Tolerant Operation of a Logical Qubit in a Diamond Quantum Processor, arXiv:2108.01646.

[96] K. Singer, U. Poschinger, M. Murphy, P. Ivanov, F. Ziesel, T. Calarco, and F. Schmidt-Kaler, Colloquium: Trapped Ions as Quantum Bits: Essential Numerical Tools, Rev. Mod. Phys. 82, 2609 (2010).

[97] T. Ruster, C. Warschburger, H. Kaufmann, C. T. Schmiegelow, A. Walther, M. Hettrich, A. Pfister, V. Kaushal, F. Schmidt-Kaler, and U. G. Poschinger, Experimental Realization of Fast Ion Separation in Segmented Paul Traps, Phys. Rev. A 90, 033410 (2014). 